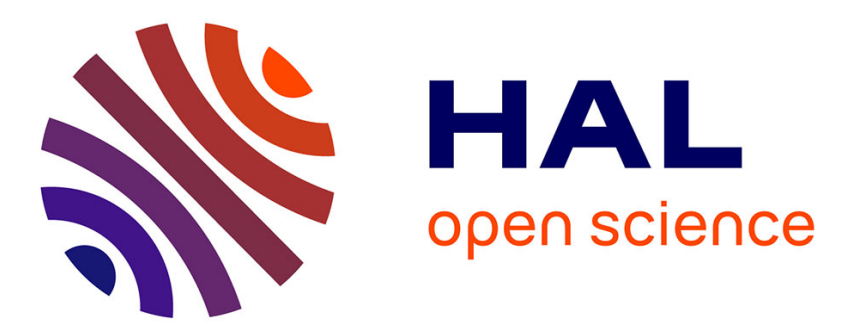

\title{
Carrier Phase Recovery for Optical Coherent M-QAM Communication Systems Using Harmonic Decomposition-based Maximum Loglikelihood Estimators
}

Trung Hien Nguyen, Michel Joindot, Pascal Scalart, Mathilde Gay, Laurent Bramerie, Olivier Sentieys, Jean-Claude Simon

\section{To cite this version:}

Trung Hien Nguyen, Michel Joindot, Pascal Scalart, Mathilde Gay, Laurent Bramerie, et al.. Carrier Phase Recovery for Optical Coherent M-QAM Communication Systems Using Harmonic Decomposition-based Maximum Loglikelihood Estimators. OSA Advanced Photonics Congress 2015 (APC 2015), Jun 2015, Boston, MA, United States. SpT4D.3, 10.1364/SPPCOM.2015.SpT4D.3 . hal-01315770

\author{
HAL Id: hal-01315770 \\ https://hal.science/hal-01315770
}

Submitted on 13 May 2016

HAL is a multi-disciplinary open access archive for the deposit and dissemination of scientific research documents, whether they are published or not. The documents may come from teaching and research institutions in France or abroad, or from public or private research centers.
L'archive ouverte pluridisciplinaire $\mathbf{H A L}$, est destinée au dépôt et à la diffusion de documents scientifiques de niveau recherche, publiés ou non, émanant des établissements d'enseignement et de recherche français ou étrangers, des laboratoires publics ou privés. 


\title{
Carrier Phase Recovery for Optical Coherent $M$-QAM Communication Systems Using Harmonic Decomposition- based Maximum Loglikelihood Estimators
}

\author{
Trung-Hien Nguyen ${ }^{1}$, Michel Joindot ${ }^{1}$, Pascal Scalart ${ }^{2}$, Mathilde Gay ${ }^{1}$, Laurent Bramerie ${ }^{1}$, Olivier Sentieys ${ }^{2}$ \\ and Jean-Claude Simon ${ }^{1}$ \\ (1) FOTON Laboratory, CNRS, University of Rennes 1, ENSSAT, F-22305 Lannion, France \\ (2) INRIA / IRISA, Campus de Beaulieu, F-35000 Rennes, France \\ E-mail: Trung-Hien.Nguyen@enssat.fr
}

\begin{abstract}
A novel feedforward $M$-QAM carrier phase recovery is presented and validated with both square and cross $M-\mathrm{QAM}$ up to 128-QAM. The proposed method outperforms the VVMPE-ML method with reduced computational effort and improved linewidth tolerance.

OCIS codes: (060.1660) Coherent communications; (060.4080) Modulation; (060.2360) Fiber optics links and subsystems.
\end{abstract}

\section{Introduction}

The demand for very high data rates in next generation optical networks has induced a renewed interest for coherent detection and advanced modulation formats such as $M$-ary quadrature amplitude modulation (M-QAM) [1]. Carrier phase recovery (CPR) is an indispensible function in such coherent receivers, in which the random phase shifts induced by laser phase noise at both transmitter and receiver sides need to be compensated. In real implementations, huge parallelization is required for feedback CPR for $100 \mathrm{~Gb} / \mathrm{s}$ or higher data rate transmission. Blind feedforward CPR techniques are more suited for practical implementation since they do not require a feedback loop [1]. Several feedforward CPR methods have been proposed so far, including the family of minimum distance blind-phase-search (BPS) methods [1], however at the cost of a high complexity. Modified BPS versions targeting complexity reduction and/or performance improvement [2 and references therein] have also been proposed. Another CPR family based on QPSK partitioning [3 and references therein] could increase the complexity when applied to high levels of $M-\mathrm{QAM}$. Furthermore, its performance is degraded when working with cross $M$-QAM. The Viterbi-Viterbi monomial-based and maximum likelihood estimator (VVMPE-ML) was proposed as an alternative candidate for CPR [4] with significant complexity reduction in comparison to the BPS method by using the power operator to weight the symbol amplitudes. This weighting method improves the CPR estimator, albeit with an increased computational effort. Moreover, adding a maximum likelihood (ML) estimator as a second stage allows refining the recovered constellation, leading to an improved estimation [4]. However, the possibility to apply VVMPE-ML to cross $M-\mathrm{QAM}$ signals has not been discussed so far.

In this paper, we propose an improved blind CPR method that utilizes the circular harmonic expansion (CHE) of loglikelihood functions (LLFs) [5] and combines a maximum likelihood estimator with the CHE method in order to refine the constellation as in VVMPE-ML. Moreover, the use of a look-up table (LUT) based on optimum weighting functions in our proposed CPR distinguishes our method (CHE-ML) from the VVMPE-ML method, resulting in not only a better laser phase noise estimation but also a reduction in overall computational effort. The CHE method alone has been studied for $M-\mathrm{QAM}$ in [5], but is, to the best of our knowledge, explored here for the first time in the context of optical coherent communication systems with a potential for efficient hardware implementation and good laser linewidth tolerance. The proposed method is numerically investigated with respect to linewidth tolerance for cross and square $M-$ QAM up to 128-QAM, showing its compatibility with the use of external cavity lasers (ECLs) with linewidths up to $70 \mathrm{kHz}$ (which is typical of commercial ECLs) for 40 GBaud signals.

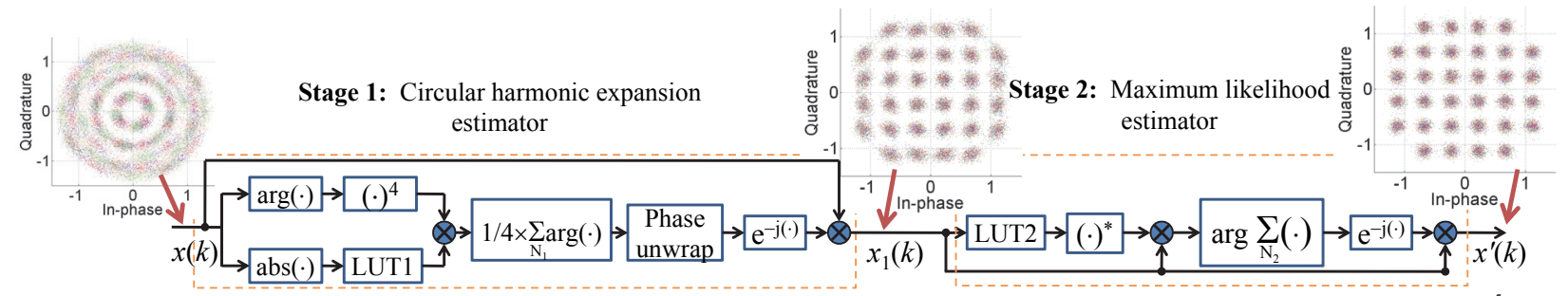

Fig. 1. CHE-ML based CPR configuration. The insets show 32-QAM constellations at each stage with $\mathrm{SNR}=20.8 \mathrm{~dB}$ and $\Delta \mathrm{v} \cdot T_{B}=10^{-5}$, demonstrating the refined constellation after second stage. 


\section{Method description}

Fig. 1 presents the configuration for $M-$ QAM CPR based on CHE-ML [5]. The idea of approximating the LLF by expanding it in series and retaining only the most significant terms results in a radius weighting function for the estimation of phase shifts and rotations. It is assumed that the received samples at the baud rate, $x(k)=r(k) \cdot \exp (j \cdot \phi(k))$, are corrupted by complex discrete additive white Gaussian noise (AWGN) with variance $\sigma^{2}$ for both real and imaginary parts. In this work, the unknown phase shift due to the laser phase noise is studied regardless of the carrier frequency offset (assumed to be completely compensated elsewhere). The LLF for this unknown phase shift is expressed as follows

$$
L L F(\phi(k) \mid x(k))=\log \left[\frac{1}{2 \pi \sigma^{2} M} \sum_{m=1}^{M} \exp \left(-\frac{\left|x(k) e^{-j \phi(k)}-C_{m}\right|^{2}}{2 \sigma^{2}}\right)\right]
$$

where $M$ is the constellation size and $C_{m}$ denotes the ideal value on the constellation for $m=1, \ldots, M[5]$.

Expanding (1) in Fourier series along the phase $\varphi$, the LLF approximation retaining the first nonzero harmonic component over $N$ symbols is given by

$$
L L F(\phi(k) \mid x(k)) \approx \operatorname{Re}\left[e^{-j 4 \phi(k)} \sum_{k=1}^{N} A_{4}(r(k)) e^{-j 4 \varphi(k)}\right]=\operatorname{Re}\left[e^{-j 4 \phi(k)} F_{4}(\{x(k)\})\right]
$$

in which $A_{4}(r)$ is the amplitude of the fourth harmonic [5]. This weighting function implemented in LUT1 is utilized for $\mathrm{CPR}$, leading to reduced computational effort compared to the use of a weighted power operator, as in the VVMPE-ML method.

Two steps are carried out for CPR, as illustrated in Fig. 1. The first step is the determination of the phase noise estimator from $N_{1}$ symbols based on the rule $\hat{\phi}=\frac{1}{4} \arg F_{4}(\{x(k)\})$. The output symbols $x_{1}(k)$ are fed to the second step consisting of the ML operation carried out on $N_{2}$ symbols to refine the phase estimation. The ML phase is calculated according to $\widehat{\phi_{M L}}=\arg \left[\sum_{k=1}^{N_{2}} D D\left(x_{1}(k)\right)^{*} x_{1}(k)\right]$, where $D D$ is the direct-detection operation based on LUT2. The inset of Fig. 1 presents an example of application of the CHE-ML method for the CPR of a 32-QAM signal with a normalized linewidth (linewidth time symbol duration product $\left(\Delta v \cdot T_{B}\right)$ ) of $10^{-5}$.

\section{Results and discussion}

In order to evaluate the performance of the proposed CPR method, numerical simulations have been carried out with 16-, 32-, 64- and 128-QAM signals under the impact of laser phase noise originating from the laser sources. The $M$-QAM signal is generated by differentially encoding and mapping a pseudo-random binary sequence (PRBS) with length of $2^{13}-1$ onto the constellation. The phase noise caused by the laser linewidth, $\Delta v$, is modeled as a discrete time random walk $\phi_{n}=\phi_{n-1}+\Delta_{n}$, in which $\Delta_{n}$ is a Gaussian random variable with zero mean and variance $2 \pi \cdot \Delta v \cdot T_{B}$, where $T_{B}$ is the QAM symbol duration. Moreover, $\sim 130000$ symbols are transmitted and corrupted by AWGN. The noise is specified by the signal-to-noise ratio (SNR) in the electrical domain. For simplicity, only one polarization is studied in this simulation. To focus on the CPR function alone, impairments introduced by carrier frequency offset, chromatic dispersion and timing clock recovery errors are assumed to be completely compensated. In the following study, 16-, 32-, 64- and 128-QAM signals at the corresponding SNRs of $18.4 \mathrm{~dB}, 20.8 \mathrm{~dB}, 24.5 \mathrm{~dB}$ and $26.6 \mathrm{~dB}$, corresponding to $1 \mathrm{~dB}$ penalty at the hard forward-error correction (FEC) bit-error ratio (BER) limit of $10^{-3}$ are considered [6].

In the first step, the optimum block length is investigated. In order to ensure a fair comparison, only the block length of the first CPR stage of CHE-ML $\left(N_{1}\right)$ is compared to that of the VVMPE-ML and BPS methods. Numerical simulations are carried out with variable block lengths under different linewidth impacts. For 16-QAM signals, the resulting BERs obtained with normalized linewidths of $10^{-4}$ and $10^{-5}$ are shown in Fig. 2(a) and (b), respectively. For 32-QAM signals, the BERs obtained for normalized linewidths of $10^{-5}$ and $10^{-6}$ are represented in Fig. 2(c) and (d), respectively. Although the impact of phase noise is different, the optimum block lengths are almost similar. The optimum block length is defined as the shortest block length value providing the target BER. More specifically, for 16-QAM, the optimum block lengths are 24/16/16 for VVMPE-ML/CHE-ML/BPS algorithms, respectively. For 32-QAM, the VVMPE-ML algorithm is no longer working, while the optimum block lengths for CHE-ML and BPS are 60 and 20, respectively. The impact of the block length for 64- and 128-QAM is evaluated in the same way without plotting the results due to space constraints, resulting in optimum values of 70/50/40 and $-/ 220 / 100$, respectively. It is observed that the BPS algorithm rapidly converges to the minimum BER when 
increasing the block lengths for high-order $M$-QAM $(M \geq 32)$, whereas the proposed method requires fewer symbols than the VVMPE-ML method to converge to the minimum BER.
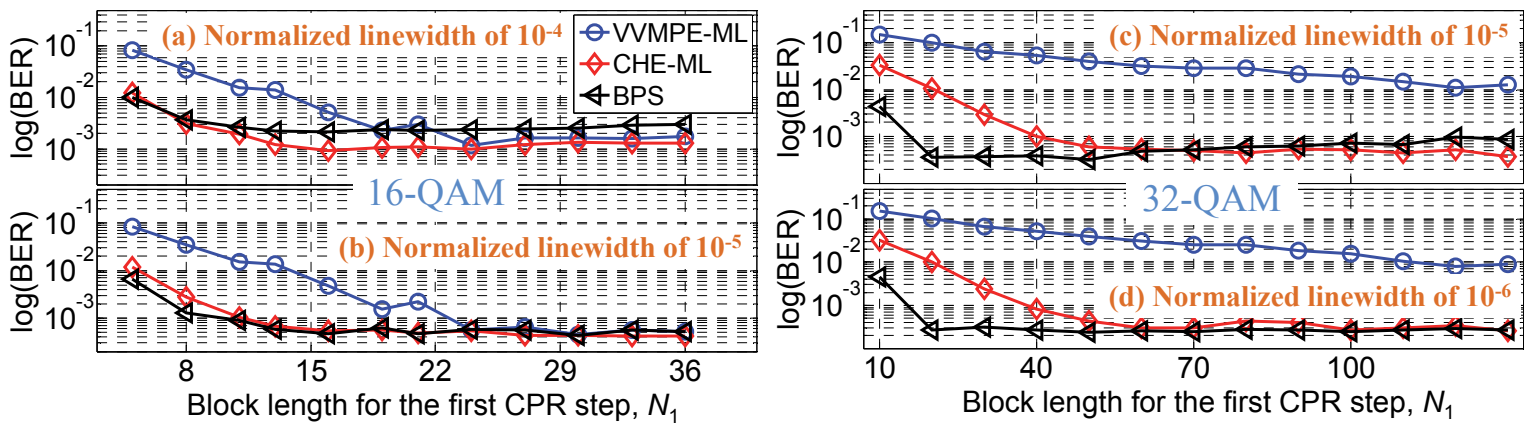

Fig. 2. Calculated BER as a function of block length for the first CPR stage for (a), (b) 16-QAM and (c), (d) 32-QAM signals.

In the next step, the laser linewidth tolerance of the CHE-ML method is compared to that of the other methods. The optimal block lengths for each method, as obtained from the previous investigation, are applied in this study. Fig. 3 presents the results of BER calculations as a function of normalized laser linewidth for 16-, 32-, 64- and 128-QAM. As expected, the BER is deteriorated when the linewidth increases. It is observed that the proposed method outperforms the VVMPE-ML method, especially with cross $M$-QAM signals, while keeping similar performance as the BPS method, which is considered as a benchmark for feedforward CPR [4]. Based on this analysis, the proposed CPR method can tolerate normalized linewidths equal to $1 \times 10^{-4}, 2.3 \times 10^{-5}, 1.3 \times 10^{-5}$, and $1.7 \times 10^{-6}$ for $16-, 32-, 64-$ and $128-\mathrm{QAM}$, respectively. At 40 GBaud, the maximum linewidth requirement to achieve $1 \mathrm{~dB}$ penalty at a BER of $10^{-3}$ is therefore $70 \mathrm{kHz}$ for 128 -QAM, which can be met using ECLs.
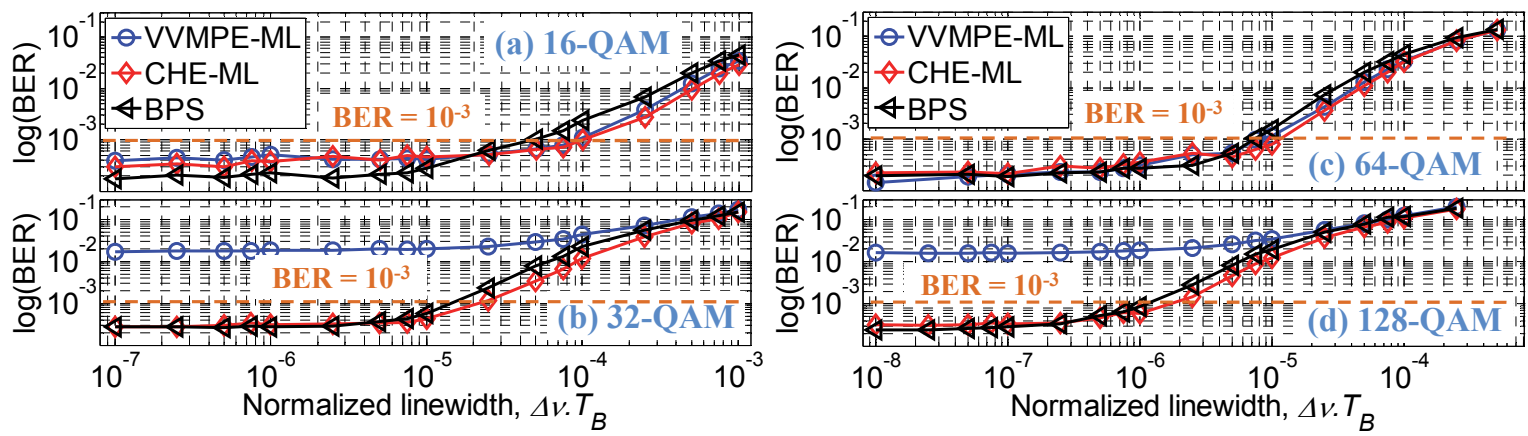

Fig. 3. Calculated BER versus linewidth for (a) 16-QAM, (b) 32-QAM, (c) 64-QAM and (d) 128-QAM signals.

In terms of complexity, our proposed method has slightly less hardware complexity than the VVMPE-ML method and benefits from reduced computational efforts thanks to the common use of LUT1 for CPR. As discussed in Ref. 4, the proposed method is also simple and presents a much lower complexity than the widely-used BPS method [1]. Although additional comparisons with other methods may be needed, it is worthy to note that CHE-ML is well suited for higher $M-\mathrm{QAM}(M>16)$ even with cross $M-\mathrm{QAM}$.

\section{Conclusions}

A simple CPR algorithm based on circular harmonic expansion is proposed and numerically validated with both square and cross $M$-QAM signals up to 128-QAM, showing its compatibility with commercial ECLs with a linewidth of $70 \mathrm{kHz}$ for 40 GBaud signals. The proposed method performance is similar to that of BPS but better than that of VVMPE-ML, with significantly reduced computational effort compared to other investigated methods.

\section{Acknowledgments}

This work was supported by the French National Research Agency (OCELOT project, ref. ANR-10-VERS-0015), the Contrat de plan Etat-Région Ponant and the French Ministry of Research. We acknowledge Christophe Peucheret for fruitful discussions.

\section{References}

[1] T. Pfau et al., "Hardware-efficient coherent digital receiver concept ...," J. Lightw. Technol. 27, 989-999 (2009).

[2] X. Zhou et al., "Low-complexity carrier phase recovery ...," IEEE Photon. Technol. Lett. 26, 1863-1866 (2014).

[3] S. M. Bilal et al., "Multi-stage CPE algorithms for 64-QAM constellations," in Proc. of OFC 2014, paper M2A.8.

[4] S. Dris et al., "M-QAM carrier phase recovery using the Viterbi-Viterbi ...," in Proc. of OFC 2013, paper OTu3I.3.

[5] A. V. Petrov et al., "Optimal blind biharmonic feedforward phase offset estimation ...," in Proc. of IEEE ICC 2013, pp. 4756-4760.

[6] T. Mizuochi, "Recent progress in forward error correction ...," IEEE J. Sel. Topics Quantum Electron. 12, 544-554 (2006). 